\title{
Infarto agudo de miocardio secundario a embolismo en una paciente con fibrilación auricular paroxística
}

Alex Gallegos Cazorlaํ, Juan Carlos More Terán²

\section{RESUMEN}

La fibrilación auricular (FA) puede producir embolismo siendo causa importante de morbilidad y mortalidad. Presentamos el caso de una paciente admitida con historia de palpitaciones, agregándose dolor retro esternal opresivo. Se encuentra FA asociada a cambios electrocardiográficos compatibles con infarto agudo de miocardio. Los biomarcadores (Troponina T y (K-MB) y los trastornos de la motilidad determinados por ecocardiografía confirmaron el diagnóstico procediéndose a la coronariografía donde se comprueba la oclusión total de arteria ventricular posterior siendo tratada exitosamente con aspiración del trombo, restableciéndose el flujo. Mientras que la FA puede encontrarse comúnmente asociada con el infarto agudo de miocardio, el tromboembolismo secundario a la FA que ocasione la oclusión coronaria total no es frecuente. Se debe considerar este escenario antes de la colocación de stents.

(Horiz Med 2015; 15(2): 66-69)

Palabras clave: Fibrilación auricular, Infarto de miocardio, Tromboembolia. (Fuente: DeCS BIREME).

Acute myocardial infarction after embolism in a patient with paroxysmal atrial fibrillation

\section{ABSTRACT}

Atrial fibrillation (AF) can produce embolism and remains as an important cause of morbidity and mortality. We report the case of a patient admitted with a history of palpitations and oppressive retro sternal pain. AF was found associated with ECG changes compatible with acute myocardial infarction. Biomarkers (Troponin T and CK-MB) and motility disorders assessed by echocardiography confirmed diagnosis and coronary angiography was performed. A total occlusion of posterior ventricular coronary artery branch was found, which was successfully treated with thrombus aspiration, reestablishing coronary flow. While AF can be commonly associated with acute myocardial infarction, thromboembolism secondary to AF producing total coronary occlusion is rare. This clinical scenario should be considered before stenting.

(Horiz Med 2015; 15(2): 66-69)

Key words: Atrial fibrillation, myocardial infarction, thromboembolism. (Source: MeSH NLM).

1 Médico Cardiólogo - Hospital Nacional Daniel Alcides Carrión (HNDAC) - Callao - Perú

Profesor de la Facultad de Medicina Humana de la Universidad de San Martín de Porres.

2 Médico Residente de cardiología - Universidad Nacional Mayor de San Marcos - Hospital Alcides Carrión (HNDAC) - Callao - Perú 


\section{INTRODUCCIÓN}

La fibrilación auricular (FA) es la arritmia cardiaca sostenida más frecuente en la clínica. La posibilidad no solo de producir embolias cerebrales, sino también sistémicas son sus consecuencias clínicas y pronósticas más relevantes siendo una causa muy importante de morbilidad y mortalidad.

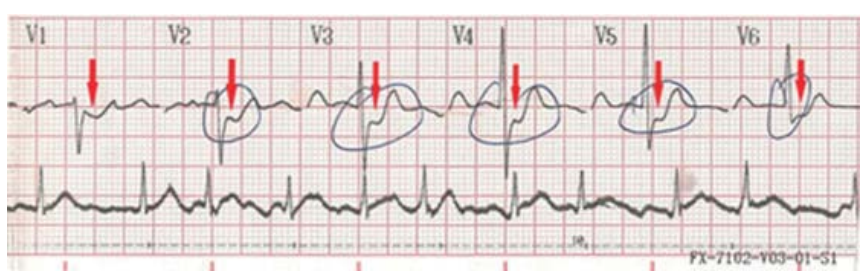

Figura 1. Electrocardiograma que muestra un ritmo de fibrilación auricular con desnivel significativo del segmento ST en derivaciones precordiales V1-V6 compatibles con imagen en espejo infarto póstero lateral STE.

Los pacientes con FA tienen de 4 a 5 veces el riesgo de stroke y cerca del doble del riesgo de morir en comparación con aquellos sin FA (1).

El infarto agudo de miocardio (IMA) es un factor de riesgo establecido para la FA (2), presentándose en un $6 \%$ a $21 \%$ de pacientes con IM (3). Por otro lado, se han reportado casos esporádicos de IMA tromboembólico en pacientes con FA $(4,5)$. Ello sugiere que la FA podría ser también un riesgo independiente de IMA.

\section{CASO CLÍNICO}

Paciente mujer de 74 años, con antecedentes de hipertensión arterial, stroke isquémico e hipertiroidismo. Admitida en la emergencia por presentar palpitaciones una hora antes de ingreso al cual se agrega dolor retro esternal opresivo 5/10. Examen físico: Frecuencia cardiaca 117 lpm, frecuencia respiratoria $20 \mathrm{rpm}$. Presión arterial $160 / 80 \mathrm{mmHg}$. Sin Ingurgitación Yugular, Bocio presente.

Murmullo vesicular de buen pasaje, sin estertores. Ruidos cardiacos arrítmicos de buena intensidad, sin soplos audibles. Pulsos simétricos, deficitarios. No edemas. Exámenes de laboratorio: Troponina T: $0,108 \mathrm{ngr} / \mathrm{ml}$ (Valor Normal< 0,003- 0,014), CK-MB 8,03 $\mathrm{ngr} / \mathrm{ml}$ (VN 1,39-6,22 $\mathrm{ngr} / \mathrm{ml}$ ).
Electrocardiograma: Fibrilación auricular, respuesta ventricular alta. Segmento ST deprimido 1-2 mm en derivación V1, V2, V3, V4, V5 y V6 (Figura 1).

La ecocardiografía muestra hipoquinesia posterolateral con fracción de eyección del $48 \%$. Diagnóstico:IMA ST no elevado (IAMSTNE).

Se administra tratamiento con aspirina, clopidogrel, enoxaparina, propranolol y nitroglicerina. La paciente continúa dolor precordial, realizándose una angiografía hallándose: Coronaria derecha dominante.

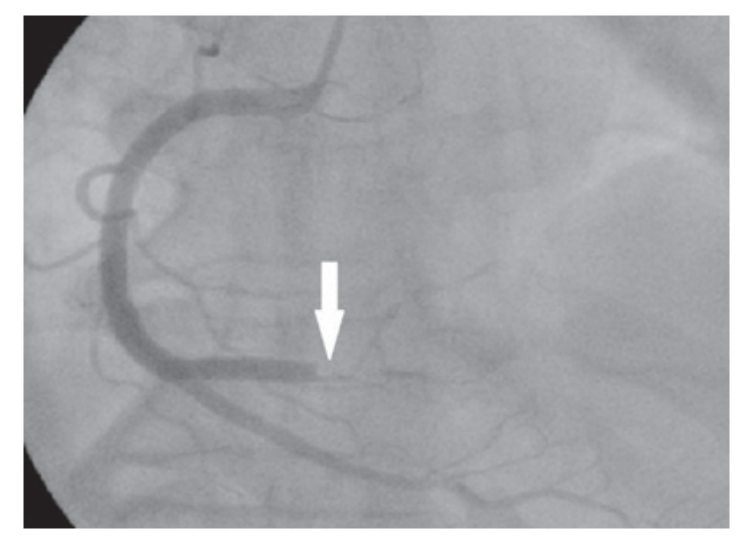

Figura 2. Coronariografía que muestra la obstrucción total del ramo ventricular posterior de la arteria coronaria derecha.

Oclusión total aguda de ramo ventricular posterior en tercio proximal (Figura 2). Se realiza angioplastia con balón y aspiración de trombo recuperándose flujo TIMI 3 (Figura 3).

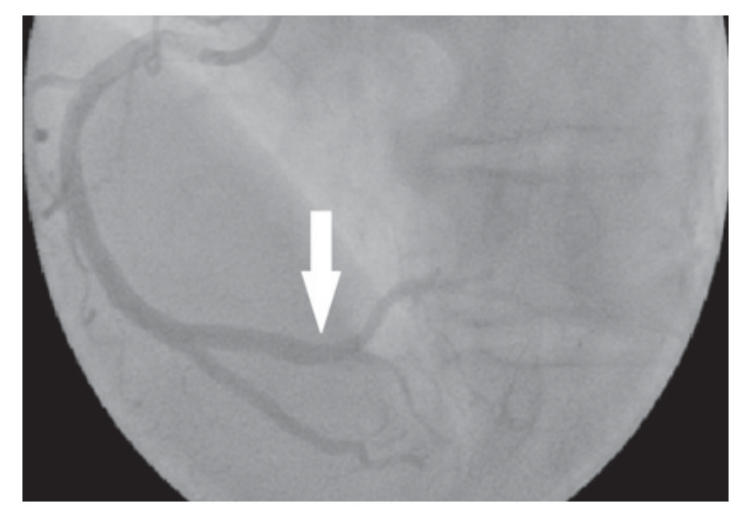

Figura 3. Coronariografía que muestra flujo restablecido TIMI 3 en el ramo ventricular posterior luego de la angioplastia y trombo aspiración. 


\section{DISCUSIÓN}

El infarto agudo de miocardio con elevación del segmento ST (IAMSTE) relacionado al embolismo de las arterias coronarias se ha descrito como raro con una prevalencia desconocida.

La infrecuencia de este suceso se ha relacionado entre otras causas con:

(a) La discrepancia entre el calibre de la aorta y el de las arterias coronarias,

(b) La localización de las arterias coronarias en la raíz de la aorta inmediatamente detrás de la válvula aórtica y

(c) El hecho que el Ilenado coronario se da en diástole (6).

Los trombos intra auriculares se desarrollan mayormente en los pacientes con FA paroxística o permanente (7) y solo se han reportado casos aislados de IMASTE en pacientes con FA causados por embolismo directo de trombos intra auriculares (8).

Nuestra paciente presentó IMSTE dentro del contexto de FA paroxística, los hallazgos electrocardiográficos inicialmente fueron interpretados como un IMSTNE por la depresión del segmento ST en múltiples derivaciones anteriores.

Ello motivó que la trombolisis no fuera utilizada de inmediato procediéndose a la angiografía coronaria por la persistencia de los síntomas.

Los hallazgos muestran la presencia de coágulo obstructivo total de la arteria coronaria derecha (CD) en su ramo ventricular posterior, de allí la presencia del segmento ST deprimido como espejo de la elevación ST en la cara posterior del ventrículo izquierdo.

Se realizó una trombo aspiración durante el procedimiento con la restauración del flujo coronario TIMI 3 sin presencia de lesiones ateromatosas significativas. Los constituyentes principales del trombo que se producen en las venas y las cámaras cardiacas son los glóbulos rojos y fibrina, por lo tanto su apariencia macroscópica es roja.
Debido a estos hallazgos en el material aspirado (Figura 4) que mostró la acumulación de glóbulos rojos y fibrina podemos conjeturar que el IMA fue causado por embolismo coronario complicando la FA.

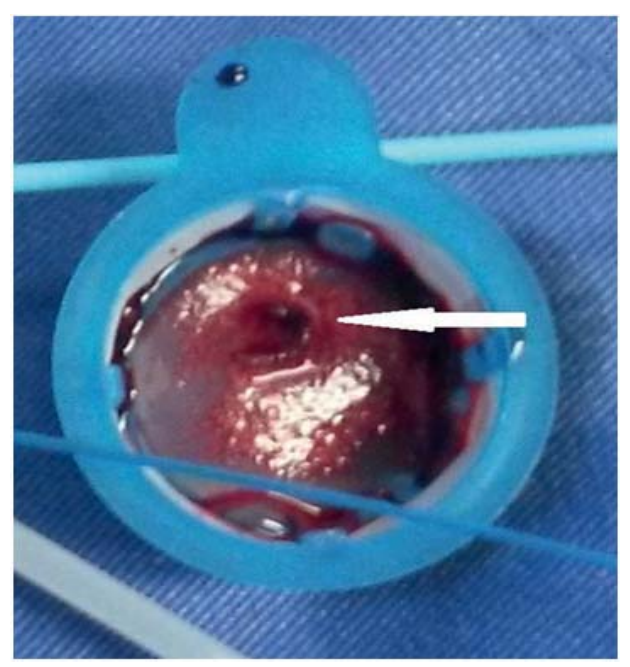

Figura 4. Trombo aspirado de la arteria ventricular posterior.

Similares hallazgosa los nuestros han sido reportados con la trombo aspiración como tratamiento exitoso (9).

Aunque el embolismo arterial coronario es reconocido, existe poca información morfológica que indique la causa del infarto de miocardio. Un estudio reveló que los pacientes con infartos embólicos arteriales representaron el 13\% de las autopsias. La mayoría de los émbolos afectaron la arteria coronaria izquierda con infartos usualmente transmurales (10).

Nuestro caso presentó infarto afectando la coronaria derecha, lo que lo hace más inusual.

Debido a su localización distal los émbolos coronarios pueden causar infartos con arterias coronaria angiográficamente normales. La embolia coronaria puede producir signos y síntomas indistinguibles de la enfermedad coronaria ateromatosa y alojarse en arterias previamente normales (10). 


\section{REFERENCIAS BIBLIOGRÁFICAS}

1. Benjamin EJ, Wolf PA, D'Agostino RB, Silbershatz $H$, Kannel WB, Levy D. Impact of atrial fibrillation on the risk of death: the Framingham Heart Study. Circulation. 1998;98(10):946-952.

2. Benjamin EJ, Chen PS, Bild DE, et al. Prevention of atrial fibrillation: report from a national heart, lung, and blood institute workshop. Circulation. 2009;119(4):606-618.

3. Schmitt J, Duray G, Gersh BJ, Hohnloser SH. Atrial fibrillation in acute myocardial infarction: a systematic review of the incidence, clinical features and prognostic implications. Eur Heart J. 2009 Garg RK, Jolly N.

4. Acute myocardial infarction secondary to thromboembolism in a patient with atrial fibrillation. Int J Cardiol. 2007;123(1):e18-e20.

5. Sakai K, Inoue K, Nobuyoshi M. Aspiration thrombectomy of a massive thrombotic embolus in acute myocardial infarction caused by coronary embolism. Int Heart J. 2007;48(3):387-392.

6. Cheng JTO, Cahill WJ. Coronary embolism. JAMA 1953;153:211-3.

7. Lam YY, Ma TK. Alternatives to chronic warfarin therapy for the preventionof stroke in patients with atrial fibrillation. Int J Cardiol 2011;150:4-11.

8. Garg RK. Acute myocardial infarction secondary to thromboembolism in a patient with atrial fibrillation. Int J Cardiol 2007;123:e18-20.

9. Sakai K1, Inoue K, Nobuyoshi M. Aspiration thrombectomy of a massive thrombotic embolus in acute myocardial infarction caused by coronary embolism. Int Heart J. 2007 May;48(3):387-92

10. Prizel KR, Hutchins GM, Bulkley BH. Coronary artery embolism and myocardial infarction. Ann InternMed. 1978 Feb;88(2):155-61.

\section{Fuentes de financiamiento}

El estudio ha sido autofinanciado por el autor.

\section{Conflictos de interés}

El autor declara no tener ningún conflicto de interés

\title{
Correspondencia:
}

\author{
Alex Gallegos Cazorla \\ Dirección: Av. La Marina 554, La Perla - Callao \\ Teléfono: 999409630 \\ Correo electrónico: gallegoscazorla@hotmail.com
}

Recibido: 24 de Enero de 2015 Aprobado: 13 de Abril de 2015 